Ann. Biol. anim. Bioch. Biophys., I969, 9 (2), I9I-204.

\title{
INFLUENCE DU REGIME MATERNEL SUR LA CROISSANCE DU JEUNE POUSSIN. EFFETS D'UNE DÉFICIENCE EN ACIDE LINOLEIQUE
}

\author{
B. LECLERCQ, J.-C. BLUM et P. DELPECH \\ avec la collaboration technique de M.-Rose SALIchon et Annette Dujon \\ Station de Recherches avicoles, \\ Centre national de Recherches zootechniques, 78 - Jouy-en-Josas \\ Institut national de la Recherche agronomique
}

\section{SOMMAIRE}

Lorsque la poule reçoit, depuis sa naissance, un régime déficient en acide linoléique, le poids de l'œuf et du nouveau-né sont également diminués d'environ Io p. Ioo. L'éclosivité est faible, le développement post-natal est considérablement retardé ; un apport de $\mathrm{x}, \mathrm{I}$ p. I 00 d'acide linoléique dans le régime du poussin ne permet pas de rétablir une croissance normale : à 28 jours, le poids des animaux est inférieur de $22 \mathrm{p}$. 100 à celui des témoins alimentés avec le même régime, mais provenant de mères qui reçoivent une quantité convenable d'acide linoléique. A l'inverse, lorsqu'on distribue un régime déficient à des poussins nés d'une mère alimentée normalement, la croissance est à peine ralentie. Dans les limites de notre étude ( 28 jours d'expérience), le développement pondéral dépend donc davantage du régime de la mère que de celui du poussin.

Lors de déficience en acide linoléique, la résorption du sac vitellin est ralentie pendant la vie embryonnaire; au cours de la vie post-natale, elle dépend de la richesse du régime en acides gras essentiels. La séparation des constituants corporels et l'analyse chimique globale montrent que la croissance reste harmonieuse quels que soient les régimes de la mère et du poussin. Les proportions de protéines et de cendres dosées dans la dépouille ne dépendent que de l'âge de l'animal. Par contre, l'aptitude à l'engraissement paraît liée à la richesse du régime maternel en acide linoléique. Quelle que soit leur alimentation, les animaux nés d'une mère recevant un régime déficient en acides gras essentiels demeurent plus maigres que les témoins pendant les 28 jours d'expérience.

Au moment de l'éclosion, la composition en acides gras de tous les tissus est étroitement liée à celle du régime maternel. Dons le foie et la carcasse, le renouvellement est rapide ; à l'âge de I4 jours, la proportion des divers acides gras est sous la seule dépendance de l'aliment ingéré par le poussin. En revanche, les lipides du cerveau demeurent beaucoup plus longtemps " marqués " par la composition du régime maternel ; à l'âge de 28 jours, cet effet est encore très accentué.

\section{INTRODUCTION}

On sait depuis longtemps que la nature des acides gras du régime de la Poule est susceptible de nodifier profondément celle de l'œuf. Des travaux plus récents ont montré qu'à leur tour, ces modifications ont un effet sur la composition des lipides 
TABLEAU $T$

Composition des régimes distribués aux poules reproductrices

\begin{tabular}{|c|c|c|c|c|}
\hline & Lot 1 & Lot 2 & Lot 3 & Lot 4 \\
\hline $\begin{array}{l}\text { Lait écrémé Spray } \ldots \ldots \\
\text { Tourteau d'arachide } \ldots \ldots \\
\text { Gélatine } \ldots \ldots \ldots \ldots \ldots \ldots \ldots \\
\text { Cellulose } \ldots \ldots \ldots \ldots \ldots \\
\text { Cérélose } \ldots \ldots \ldots \ldots \ldots \ldots \\
\text { Huile de maĩs } \ldots \ldots \ldots \ldots \\
\text { Carbonate de calcium } \ldots \\
\text { Phosphate bicalcique } \ldots \ldots \\
\text { DL-méthionine } \ldots \ldots \ldots \\
\text { Minéraux et vitamines ... }\end{array}$ & $\begin{array}{r}27,00 \\
5,00 \\
5,00 \\
5,00 \\
51,00 \\
0 \\
5,00 \\
1,50 \\
0,01 \\
0,45\end{array}$ & $\begin{array}{r}27,00 \\
5,00 \\
5,00 \\
7,00 \\
47,00 \\
2,00 \\
5,00 \\
1,50 \\
0,01 \\
0,45\end{array}$ & $\begin{array}{r}27,00 \\
5,00 \\
5,00 \\
9,00 \\
43,00 \\
4,00 \\
5,00 \\
1,50 \\
0,01 \\
0,45\end{array}$ & $\begin{array}{r}27,00 \\
5,00 \\
5,00 \\
15,00 \\
31,00 \\
10,00 \\
5,00 \\
1,50 \\
0,01 \\
0,45\end{array}$ \\
\hline 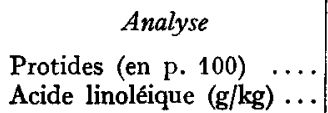 & $\begin{array}{c}15,6 \\
0,330\end{array}$ & $\begin{array}{l}15,6 \\
10,330\end{array}$ & $\begin{array}{l}15,6 \\
20,330\end{array}$ & $\begin{array}{l}15,6 \\
\mathbf{5 0 , 3 3 0}\end{array}$ \\
\hline
\end{tabular}

Complément minéral et vitaminique (p. $100 \mathrm{~kg}$ d’aliment) : vitamine A : $800000 \mathrm{UI}$, vitamine $\mathrm{D}_{3}$ $100000 \mathrm{UI}$, vitamine $\mathrm{K}: 0,1 \mathrm{~g}$, tocophérol à $25 \%: 16 \mathrm{~g}$, thiamine $: 0,3 \mathrm{~g}$, riboflavine $: 0,3 \mathrm{~g}$, pyridoxine : $0,5 \mathrm{~g}$, cyanocobalamine : $0,01 \mathrm{~g}$, pantothénate de calcium : $0,5 \mathrm{~g}$, biotine $: 0,1 \mathrm{~g}$, acide folique : $0,1 \mathrm{~g}$, BHT : $10 \mathrm{~g}$, choline à $25 \%: 200 \mathrm{~g}$, chlorure de sodium : $150 \mathrm{~g}$, sulfate de manganèse : $10 \mathrm{~g}$, citrate de fer $: 5 \mathrm{~g}$, iodure de potassium $: 0,5 \mathrm{~g}$.

TABLEAU 2

Composition des régimes distribués aux poussins

\begin{tabular}{|c|c|c|}
\hline Matière première (p. 100) & Aliment carencé & Aliment normal \\
\hline Tourteau d'arachide ........... & 24,00 & 24,00 \\
\hline Lait écrémé Spray . ........... & 20,00 & 20,00 \\
\hline Gélatine $\ldots \ldots \ldots \ldots \ldots \ldots \ldots \ldots$ & 2,00 & 2,00 \\
\hline Huile de maïs $\ldots \ldots \ldots \ldots \ldots \ldots$ & 0,00 & 2,00 \\
\hline Cellulose $\ldots \ldots \ldots \ldots \ldots \ldots \ldots$ & 2,30 & 5,00 \\
\hline Cérélose $\ldots \ldots \ldots \ldots \ldots \ldots \ldots$ & 46,70 & 42,00 \\
\hline L-Lysine $\ldots \ldots \ldots \ldots \ldots \ldots \ldots$ & 0,50 & 0,50 \\
\hline DL-Méthionine $\ldots \ldots \ldots \ldots \ldots \ldots$ & 0,25 & 0,25 \\
\hline Mélange minéral (1) . .......... & 2,65 & 2,65 \\
\hline Mélange vitaminique $\left({ }^{2}\right) \ldots \ldots \ldots$ & 1,60 & 1,60 \\
\hline Taux protidique (p. 100) $\left(^{(3)}-\ldots\right.$ & 21,10 & 21,10 \\
\hline Teneur en acide linoléïque $(\mathrm{g} / \mathrm{kg})$ & 1,01 & 11,00 \\
\hline & & \\
\hline
\end{tabular}

( $\left.{ }^{1}\right)$ Mélange minéral (pour $100 \mathrm{~kg}$ ) : carbonate de calcium : $600 \mathrm{~g}$, phosphate monopotassique : $435 \mathrm{~g}$, chlorure de sodium : $300 \mathrm{~g}$, sulfate de magnésium : $300 \mathrm{~g}$, phophate bicalcique : $1000 \mathrm{~g}$, sulfate de manganèse : $10 \mathrm{~g}$, citrate de fer $: 6 \mathrm{~g}$, sulfate de zinc : $2 \mathrm{~g}$, sulfate de cuivre : $0,5 \mathrm{~g}$, iodure de potassium : $0,5 \mathrm{~g}$.

(2) mélange vitaminique (pour $100 \mathrm{~kg}$ ) : vitamine A : $800000 \mathrm{UI}$, vitamine $\mathrm{D}_{3}: 100000 \mathrm{UI}$, vitamine $\mathrm{K}: 0,1 \mathrm{~g}$, tocophérol à $25 \%: 16 \mathrm{~g}$, thiamine : $0,3 \mathrm{~g}$, pyridoxine : $0,5 \mathrm{~g}$, cyanocobalamine : $0,01 \mathrm{~g}$, pantothénate de calcium : $0,5 \mathrm{~g}$, biotine : $0,1 \mathrm{~g}$, acide folique : $0,1 \mathrm{~g}, \mathrm{BHT}: 10 \mathrm{~g}$, choline à $25 \%: 1400 \mathrm{~g}$.

(3) $\mathrm{N} \times 6,25$. 
tissulaires du Poussin au moment de l'éclosion (ISAAKs et al., I964 ; LECLERCQ, I966 ; DoNALDSON, I967). Lorsque le régime maternel contient une quantité insuffisante d'acide linoléique, les graisses du nouveau-né sont pauvres en cet acide gras essentiel (MENGE et al., I964). Dès lors, on peut se demander si cette déficience influence la vie post-natale et quel sera le plus important du régime de la mère ou du régime du poussin.

Dans une courte note, HoPkins et NESHEIM (I962) apportent une réponse partielle à cette question. Lres poussins nés d'une mère carencée en acide linoléique croissent plus lentement que les animaux témoins; une addition d'huile de soja au régime du poussin permet de pallier ce défaut. Cependant, les auteurs n'indiquent pas la composition exacte de leurs régimes et ils ne comparent pas l'effet d'une déficience suivant qu'elle survient avant (régime maternel) ou après la naissance (régime du poussin). Il nous a paru utile de reprendre cette étude en cherchant à préciser l'effet sur la croissance post-natale et l'importance relative des régimes de la mère et du poussin. En outre, pour essayer de comprendre comment intervient la déficience en acide linoléique, nous avons effectué diverses analyses portant sur la composition corporelle et la répartition des acides gras dans les tissus du Poussin entre o et 28 jours.

\section{MATÉRIEL E'T MÉTHODES}

\section{a) Animaux et régimes}

Depuis leur naissance jusqu'à l'âge de I 8 semaines, 50 poulettes d'une souche Cornish reçoivent un régime contenant moins de 0,05 p. Ioo d'acide linoélique. Elles sont alors réparties en 4 lots de I2 poules selon la teneur du régime en acide linoléique ajouté : $0, \mathrm{I}, 2$ ou $5 \mathrm{p}$. Io०. La composition exacte de ces régimes est indiquée dans le tableau $\mathrm{I}$.

Lorsque la période de ponte est assez avancée pour que le poids des œufs dépasse $5 \circ$ grammes dans tous les lots, on insémine chaque poule 2 fois par semaine avec un mélange de spermes de coqs de la même souche Cornish. Les oufs pondus au cours d'une même semaine sont placés dans un incubateur et isolés dans des cases individuelles au moment de l'éclosion. Les poussins ainsi identifiés sont élevés par groupe de 3 dans des batteries de cages chauffées électriquement. On leur distribue ad libitum l'un des deux régimes dont la composition est indiquée dans le tableau 2.

L'aliment normal et l'aliment lipidoprive ne diffèrent que par leurs teneurs en huile de maïs, cellulose et cérélose. Ils restent isoénergétiques mais renferment une quantité différente d'acide linoléique : 0,1 ou I,I p. 100 ; ils sont, par ailleurs, complets et équilibrés.

En tenant compte de leur origine, les animaux sont répartis en 8 lots : 4 régimes maternels $\times 2$ régimes de croissance. On effectue 6 séries d'incubation d'œufs pondus au cours d'une semaine. Malheureusement, par suite d'un accident d'élevage, 5 séries seulement pourront être prises en considération. En outre, l'éclosivité étant médiocre, on ne disposera pour l'expérience que de $23^{6}$ poussins répartis dans les 8 lots de la manière suivante :

\begin{tabular}{|c|c|c|c|c|}
\hline & & $\begin{array}{l}\text { Régime maternel } \\
\qquad \text { (tabl. 1) }\end{array}$ & $\begin{array}{l}\text { Régime des poussins } \\
\text { (tabl. 2) }\end{array}$ & $\begin{array}{l}\text { Nombre de poussins } \\
\text { mis en expérience }\end{array}$ \\
\hline $\begin{array}{l}\text { Lot } 1 \mathrm{C} \\
\text { Lot } 1 \mathrm{~N} \\
\text { Lot } 2 \mathrm{C} \\
\text { Lot } 2 \mathrm{~N} \\
\text { Lot } 3 \mathrm{C} \\
\text { Lot } 3 \mathrm{~N} \\
\text { Lot } 4 \mathrm{C} \\
\text { Lot } 4 \mathrm{~N}\end{array}$ & $\begin{array}{l}\ldots \ldots \ldots \ldots \\
\ldots \ldots \ldots \ldots \\
\ldots \ldots \ldots \ldots \\
\ldots \ldots \ldots \ldots \\
\ldots \ldots \ldots \ldots \\
\ldots \ldots \ldots \ldots \\
\ldots \ldots \ldots \ldots \\
\text { (lot témoin) }\end{array}$ & $\begin{array}{l}1 \text { (sans lipides) } \\
1 \\
2 \text { (2\% d'huile de maîs) } \\
2 \\
3 \text { (4\% d'huile de maïs) } \\
3 \\
4 \text { (10\% d'huile de maïs) } \\
4\end{array}$ & $\begin{array}{l}\text { Lipidoprive } \\
\text { Normal } \\
\text { Lipidoprive } \\
\text { Normal } \\
\text { Lipidoprive } \\
\text { Normal } \\
\text { Lipidoprive } \\
\text { Normal }\end{array}$ & $\begin{array}{l}22 \\
24 \\
23 \\
20 \\
35 \\
35 \\
42 \\
35\end{array}$ \\
\hline
\end{tabular}


Les animaux du lot $4 \mathrm{~N}$ élevés dans de bonnes conditions et provenant des mères recevant le meilleur régime seront considérés comme témoins de référence.

Des animaux de chaque lot provenant de différentes séries sont sacrifiés au moment de l'éclosion (série 2), à l'âge de 2 jours (série 5), 4 jours (série 5 ), 7 jours (séries I, 3 et 4), 14 jours (séries 1,3 et 4), $2 \mathrm{I}$ jours (séries I et 4 ) et 28 jours (série 3 ).

Après le sacrifice, on sépare et on pèse le cerveau, le foie, la peau et les plumes, le sac vitellin et le reste de la carcasse.

\section{Composition chimique globale.}

\section{b) Méthodes analytiques}

On détermine la composition chimique globale de la dépouille (carcasse + peau et plume) : matières sèches, protéines, lipides, cendres, dans le seul cas des animaux appartenant aux lots $\mathrm{IC}, \mathrm{IN}$, ${ }_{4} \mathrm{C}$ et ${ }_{4} \mathrm{~N}$.

L'azote est dosé par la méthode Kjehldal et les lipides totaux de la carcasse par la méthode de DelpeCh et al. (1966).

Les lipides du cerveau et du foie sont extraits par le mélange chloroforme-méthanol-eau (2-I-I).

Composition en acides gras des lipides de différents tissus.

Les esters méthyliques obtenus par transméthylation sont analysés en chromatographie gazliquide sur DEGS à ${ }_{17} 6^{\circ} \mathrm{C}$. L'appareil (Proffit, France) est muni d'un détecteur à ionisation de flamme.

\section{RÉSULTATS}

\section{a) Effet du régime de la Poule sur le poids de l'auf}

Dans le tableau 3, nous montrons l'influence du régime sur le poids des œufs qui ont donné naissance à des poussins après incubation. Cette influence est appréciable ; dans chaque série, l'ouf pèse d'autant plus lourd que le régime est riche en acide linoléique. La différence observée entre le lot I (régime sans lipides) et le lot 4 (ro p. roo d'huile de mais) est de 6 à 8 grammes; elle est très significative $(P<0,0 I)$.

TABLEAU 3

\begin{tabular}{|c|c|c|c|c|c|c|c|c|c|c|}
\hline \multirow{2}{*}{ Lot } & \multicolumn{2}{|c|}{$\begin{array}{l}\text { Série d'incuba- } \\
\text { tion } n^{0} 1\end{array}$} & \multicolumn{2}{|c|}{ Série 2} & \multicolumn{2}{|c|}{ Série 3} & \multicolumn{2}{|c|}{ Série 4} & \multicolumn{2}{|c|}{ Série 5} \\
\hline & $\begin{array}{l}\text { Poids } \\
\text { moyen }\end{array}$ & $\begin{array}{l}\text { Écart- } \\
\text { type }\end{array}$ & $\begin{array}{l}\text { Poids } \\
\text { moyen }\end{array}$ & $\begin{array}{l}\text { Écart- } \\
\text { type }\end{array}$ & $\begin{array}{l}\text { Poids } \\
\text { moyen }\end{array}$ & $\begin{array}{l}\text { Ecart- } \\
\text { type }\end{array}$ & $\begin{array}{l}\text { Poids } \\
\text { moyen }\end{array}$ & $\begin{array}{l}\text { Écart- } \\
\text { type }\end{array}$ & $\begin{array}{r}\text { Poids } \\
\text { moyen }\end{array}$ & $\begin{array}{l}\text { Ecart- } \\
\text { type }\end{array}$ \\
\hline Régime lipidoprive & $48,35 * *$ & 2,90 & $49,71 * *$ & 2,27 & $49,70 * *$ & 5,10 & $51,35 * *$ & 4,21 & $52,89 * *$ & 2,56 \\
\hline $\begin{array}{l}\text { Régime à } 2 \text { p. } 100 \\
\text { d'huile de mails } \ldots \\
3\end{array}$ & 52,28 & 2,26 & 53,41 & 2,96 & 55,34 & 2,47 & 55,87 & 1,65 & 54,83 & 0,81 \\
\hline 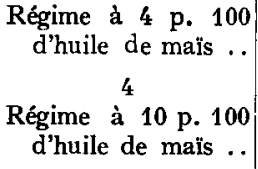 & 54,45 & 3,22 & 55,71 & 6,50 & 56,12 & 4,29 & 59,68 & 4,51 & 60,75 & 4,72 \\
\hline
\end{tabular}

* Différent du lot 4 au seuil de signification de 5 p. 100.

** Différent du lot 4 au seuil de signification de 1 p. 100. 
Il faut également remarquer que les régimes déficients en acide linoléique freinent l'augmentation du poids de l'œuf qui survient normalement à cette époque. L'écart entre les lots déficients et le lot 4 paraît de plus en plus accusé.

\section{b) Croissance des poussins}

Le tableau 4 indique à la fois le poids moyen du poussin $(\mathrm{P})$, l'écart-type $(\mathrm{S})$ et le nombre d'animaux (N) selon les lots à $0,7, I 4,21$ et 28 jours. Quelle que soit leur origine maternelle, les poussins qui reçoivent un régime carencé en acide linoléique ont une croissance plus lente que leurs congénères bénéficiant d'un aliment équilibré. Cependant, cet effet n'est marqué qu'à partir de la $3^{\mathbf{e}}$ semaine ; il concerne tout particulièrement les animaux provenant de mères recevant elles-mêmes un régime déficient en acide linoléique : à $2 \mathrm{I}$ jours, les poussins du lot I $\mathrm{C}$ ont un poids inférieur de 33 p. roo à ceux du lot I $\mathbf{N}$.

TABLEAU 4

Croissance des poussins

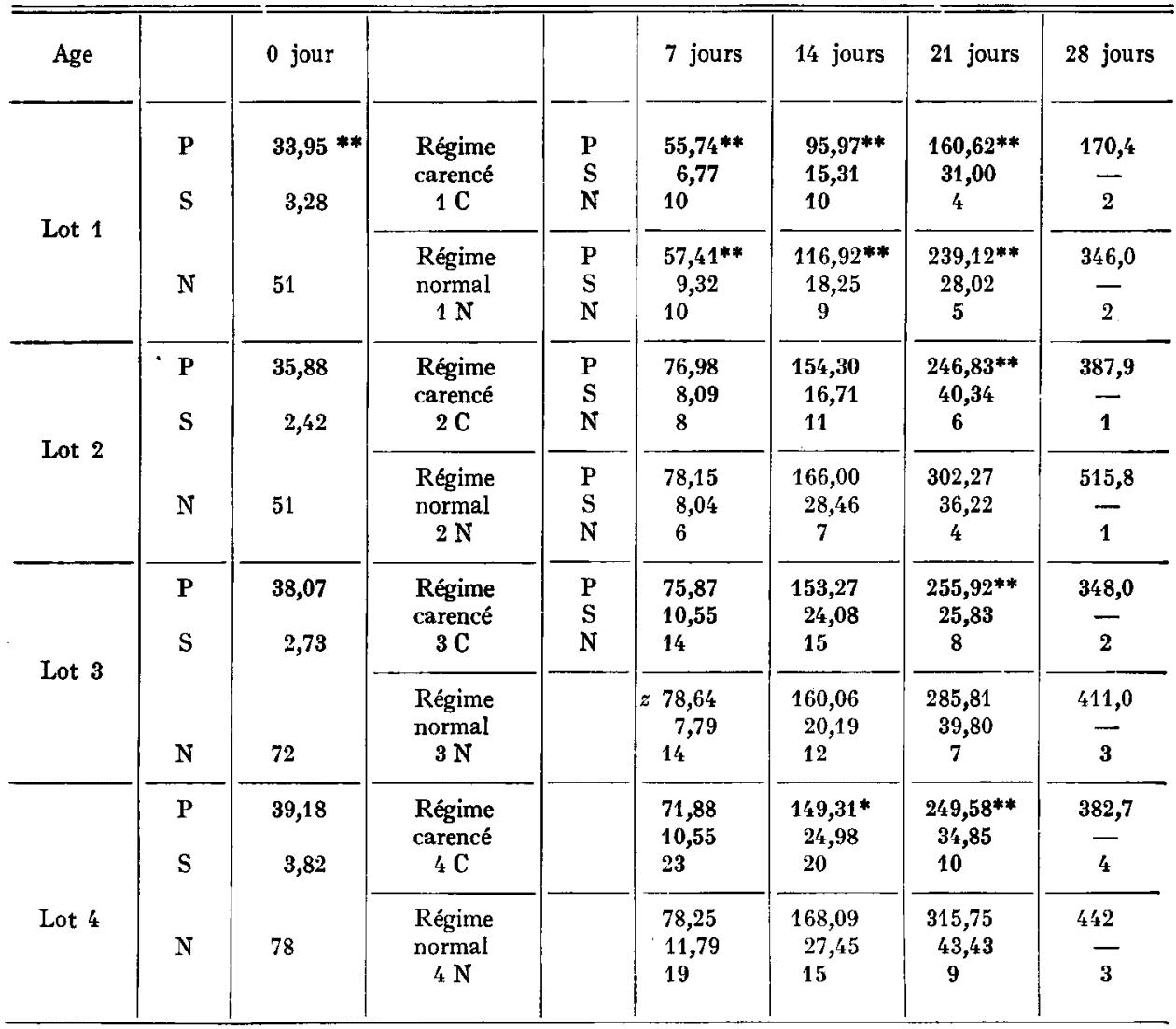

( $\mathrm{P}=$ poids moyen (en $\mathrm{g}$ ),$\quad \mathrm{S}=$ écart-type, $\quad \mathrm{N}=$ nombre de données).

* Different du lot $4 \mathrm{~N}$ au seuil de signification de 5 p. 100.

** Différent du lot $4 \mathrm{~N}$ au seuil de signification de 1 p. 100. 
Pendant les I4 premiers jours de vie, le développement pondéral dépend surtout du régime maternel. Par la suite, l'influence de la mère reste très importante et ne tend que lentement à s'estomper. Si l'on compare la croissance des animaux appartenant aux lots I $\mathrm{N}$ (régime de la mère carencé, régime du poussin normal) et $4 \mathrm{~N}$ (régimes de la mère et du poussin normaux), on constate que le poids des premiers est inférieur à celui des seconds de I7,5 p. Ioo au moment de la naissance, $26 \mathrm{p}$. IOO à 7 jours, 3 I p. IOO à I 4 jours, 24 p. IOO à 2 I jours et 22 p. IOO à 28 jours. Dans tous. les cas, les différences sont très significatives, sauf pour l'âge de 28 jours qui ne concerne que trop peu d'animaux.

\section{c) Indice de consommation}

L'indice de consommation (quantité d'aliment consommé par unité de gain de poids) témoigne dans une certaine mesure de l'efficacité alimentaire. Sa valeur a été calculée au cours de 2 périodes : o à $I_{4}$ jours et $I_{5}$ à 28 jours. Les résultats sont. consignés dans le tableau 5 .

\section{TABLEAU 5}

Valeurs de l'indice de consommation

\begin{tabular}{|c|c|c|c|c|c|c|c|c|}
\hline Lot & $1 \mathrm{C}$ & $1 \mathrm{~N}$ & $2 \mathrm{C}$ & $2 N$ & $3 \mathrm{C}$ & $3 \mathrm{~N}$ & $4 \mathrm{C}$ & $4 \mathrm{~N}$ \\
\hline $\begin{array}{l}\text { de } 0 \text { à } 14 \text { jours } \ldots . \\
\text { de } 14 \text { à } 28 \text { jours } \ldots .\end{array}$ & $\begin{array}{l}2,5 \\
3,1\end{array}$ & $\begin{array}{l}2,2 \\
2,1\end{array}$ & $\begin{array}{l}2,2 \\
2,4\end{array}$ & $\begin{array}{l}2,1 \\
2,1\end{array}$ & $\begin{array}{l}2,0 \\
2,4\end{array}$ & $\begin{array}{l}2,1 \\
2,1\end{array}$ & $\begin{array}{l}1,9 \\
2,9\end{array}$ & $\begin{array}{l}1,9 \\
2,1\end{array}$ \\
\hline
\end{tabular}

Pendant les I4 premiers jours de vie, l'indice de consommation est voisin de 2 ; il diffère peu d'un lot à l'autre; sa valeur n'augmente que très légèrement lorsque les poussins sont issus de mères recevant un régime déficient en acide linoléique. Entre le $\mathrm{I}^{\mathrm{e}}$ et $\mathrm{le} 28^{\mathrm{e}}$ jour, $1^{\prime}$ influence maternelle est négligeable; $1^{\prime}$ indice de consommation dépend seulement de la nature de l'aliment distribué aux animaux; il tend quelque peu à s'élever lors d'un apport insuffisant en acides gras essentiels.

\section{d) Données analytiques}

La séparation des constituants corporels et les différentes analyses portant sur la composition chimique des tissus concernent seulement les animaux des lots I

TABLEAU 6

Nombre d'animaux sur lesquels ont porté les analyses. effectuées au cours de la croissance

\begin{tabular}{|c|c|c|c|c|c|c|c|}
\hline Age & 0 jour & 2 jours & 5 jours & 7 jours & 14 jours & 21 jours & $i^{28 \text { jours }}$ \\
\hline 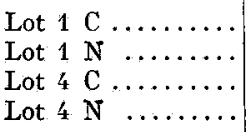 & $\frac{9}{-}$ & $\begin{array}{l}1 \\
4 \\
4 \\
2\end{array}$ & $\begin{array}{l}3 \\
3 \\
4 \\
4\end{array}$ & $\begin{array}{l}3 \\
4 \\
9 \\
7\end{array}$ & $\begin{array}{l}4 \\
4 \\
9 \\
8\end{array}$ & $\begin{array}{l}4 \\
4 \\
6 \\
6\end{array}$ & $\begin{array}{l}2 \\
1 \\
5 \\
3\end{array}$ \\
\hline
\end{tabular}


(IC, IN) et $4(4 \mathrm{C}, 4 \mathrm{~N})$, autrement dit les poussins dont le régime maternel était soit très déficient, soit très riche en acide linoléique. Dans le tableau 6 , nous indiquons le nombre d'animaux intéressés par ces analyses effectuées chez chaque individu sacrifié au cours de la croissance.

Nous interpréterons les résultats obtenus en tenant compte du nombre d'animaux analysés à chaque stade de la croissance.

ג) Constituants corporels.

Nous ne mentionnerons ni le poids du foie, ni le poids de la carcasse, ni celui de la peau et des plumes, car leurs proportions relatives sont indépendantes des régimes distribués à la mère et au poussin. Elles varient seulement en fonction de l'âge de l'animal. Les données montrant comment évolue le poids du sac vitellin après l'éclosion, retiendront davantage notre attention ; elles font l'objet du tableau 7.

\section{TABLEAU 7}

Poids du sac vitellin au moment de l'éclosion et dans les jours qui suivent

\begin{tabular}{|c|c|c|c|c|c|c|}
\hline \multirow[t]{2}{*}{ Lot } & \multicolumn{3}{|c|}{$\begin{array}{l}\text { Poids du sac vitellin (en g) } \\
\text { chez le poussin âgé de : }\end{array}$} & \multicolumn{3}{|c|}{$\begin{array}{l}\text { Valeur du rapport } \frac{\text { Poids du sac vitellin } \times 100}{\text { Poids du corps }} \\
\text { chez le poussin âgé de: }\end{array}$} \\
\hline & 0 jour & 2 jours & 5 jours & 0 jour $r$ & 2 jours & 5 jours \\
\hline \begin{tabular}{lll} 
& \multicolumn{1}{l}{$\mathrm{C}$} & $\ldots$ \\
1 & $\mathrm{~N}$ & $\ldots$ \\
4 & $\mathrm{C}$ & $\ldots$ \\
4 & $\mathrm{~N}$ & $\ldots$
\end{tabular} & $\begin{array}{l}5,8 \\
5,8 \\
5,1 \\
5,1\end{array}$ & $\begin{array}{l}1,0 \\
6,6 \\
2,1 \\
2,6\end{array}$ & $\begin{array}{l}0,1 \\
1,3 \\
0,6 \\
0,2\end{array}$ & $\begin{array}{l}17,5^{* *} \\
17,5^{* *} \\
13,4 \\
13,4\end{array}$ & $\begin{array}{c}3,2 \\
22,0^{* *} \\
4,3 \\
5,1\end{array}$ & $\begin{array}{l}0,2 \\
2,9 \\
1,0 \\
\mathbf{0 , 4}\end{array}$ \\
\hline
\end{tabular}

** Différences très significatives avec le lot $4 \mathrm{~N}(\mathrm{P}<0,01)$.

Au moment de l'éclosion, le poids du sac vitellin est plus élevé chez les poussins issus d'une mère carencée en acide linoléique. Compte tenu de la taille réduite de ces nouveau-nés, le sac vitellin représente $17,5 \mathrm{p}$. Ioo du poids du corps contre I3,4 p. Ioo chez les animaux témoins, la différence étant significative. La résorption des réserves vitellines déficientes en acide linoléique est donc ralentie au cours de l'embryogenèse ; elle l'est également et davantage encore après la naissance lorsque l'animal dispose d'un régime riche en acide linoléique : cas du lot $\mathrm{IN}$ dont le sac vitellin représente 22 p. Ioo du poids du corps 2 jours après la naissance. Par contre, chez les poussins nés d'une mére recevant un régime normal, la distribution d'un régime carencé ne modifie en rien la cinétique suivant laquelle le sac vitellin est résorbé au cours de la vie post-natale.

ß) Composition chimique globale de la dépouille (carcasse + peau et plumes).

Nous avons dosé les matières sèches, les protéines $(\mathrm{N} \times 6,25)$, les lipides et les cendres. Les résultats sont mentionnés dans le tableau 8 sous forme de teneurs moyennes exprimées en grammes par roo grammes de poids frais. 


\section{TABLEAU 8}

Composition chimique globale de la dépouille. Teneurs moyennes exprimées en g/roo de poids frais

\begin{tabular}{|c|c|c|c|c|c|c|c|c|}
\hline & \multirow{2}{*}{ Lot } & \multicolumn{7}{|c|}{ Age en jours } \\
\hline & & 0 & 2 & 5 & 7 & 14 & 21 & 28 \\
\hline \multirow{4}{*}{$\begin{array}{l}\text { Matières } \\
\text { sèches }\end{array}$} & $1 \mathrm{C}$ & $\begin{array}{c}19,7 \\
(17,3-21,5)\end{array}$ & 20,6 & $\begin{array}{c}29,4 \\
(19,9-21,1)\end{array}$ & $\begin{array}{c}23,4 \\
(20,5-24,6)\end{array}$ & $\begin{array}{c}24,4 \\
(22,0-26,6)\end{array}$ & $\begin{array}{c}26,7 \\
(24,0 \cdot 30,0)\end{array}$ & $\begin{array}{c}27,7 \\
(26,0-29,5)\end{array}$ \\
\hline & $1 \mathrm{~N}$ & - & $\begin{array}{c}19,7 \\
(18,0-21,0)\end{array}$ & $\begin{array}{c}19,7 \\
(17,0-22,0)\end{array}$ & $\begin{array}{c}23,3 \\
(22,0-25,2)\end{array}$ & $\begin{array}{c}25,0 \\
(24,0-26,5)\end{array}$ & $\begin{array}{c}27,1 \\
(26,0-28,0)\end{array}$ & 28,1 \\
\hline & $4 \mathrm{C}$ & $\begin{array}{c}21,0 \\
(18,3-22,8)\end{array}$ & $\begin{array}{c}20,8 \\
(18,8-22,3)\end{array}$ & $\begin{array}{c}20,7 \\
(20,2 \cdot 21,4)\end{array}$ & $\begin{array}{c}22,8 \\
(20,3-25,0)\end{array}$ & $\begin{array}{c}26,6 \\
(24,0-28,0)\end{array}$ & $\begin{array}{c}28,7 \\
(26,2-30,5)\end{array}$ & $\begin{array}{c}30,0 \\
(29,0-32,0)\end{array}$ \\
\hline & $4 N$ & - & $\begin{array}{c}21,5 \\
(21,0-22,0)\end{array}$ & $\begin{array}{c}23,2 \\
(21,0-22,0)\end{array}$ & $\begin{array}{c}23,4 \\
(22,0-24,4)\end{array}$ & $\begin{array}{c}26,6 \\
(25,0-28,1)\end{array}$ & $\begin{array}{c}28,5 \\
(26,0-29,9)\end{array}$ & $\begin{array}{c}28,5 \\
(28,0-29,0)\end{array}$ \\
\hline \multirow{4}{*}{$\begin{array}{l}\text { Protéines } \\
\mathrm{Nx} 6,25\end{array}$} & $1 \mathrm{C}$ & $\begin{array}{c}14,1 \\
(13,3-15,2)\end{array}$ & 15,1 & $\begin{array}{c}14,8 \\
(14,0-15,6)\end{array}$ & $\begin{array}{c}16,0 \\
(14,9-17,1)\end{array}$ & $\begin{array}{c}17,5 \\
(16,0-18,9)\end{array}$ & $\begin{array}{c}19,0 \\
(18,0-19,7)\end{array}$ & $\begin{array}{c}19,9 \\
(19,4-20,4)\end{array}$ \\
\hline & $1 \mathrm{~N}$ & - & $\begin{array}{c}15,2 \\
(13,0-17,0)\end{array}$ & $\begin{array}{c}14,3 \\
(13,0-16,0)\end{array}$ & $\begin{array}{c}16,5 \\
(16,0-17,5)\end{array}$ & $\begin{array}{c}17,6 \\
(17,0-18,0)\end{array}$ & $\begin{array}{c}18,7 \\
(18,0-19,2)\end{array}$ & 19,6 \\
\hline & $4 \mathrm{C}$ & $\begin{array}{c}14,5 \\
(13,4-15,5)\end{array}$ & $\begin{array}{c}14,7 \\
(13,6-16,0)\end{array}$ & $\begin{array}{c}14,7 \\
(13,9-15,0)\end{array}$ & $\begin{array}{c}16,4 \\
(15,0-18,0)\end{array}$ & $\begin{array}{c}17,5 \\
(16,0-18,7)\end{array}$ & $\begin{array}{c}19,4 \\
(18,8-19,9)\end{array}$ & $\begin{array}{c}19,6 \\
(19,0-20,0)\end{array}$ \\
\hline & $4 N$ & - & $\begin{array}{c}14,0 \\
(14,0-14,2)\end{array}$ & $\begin{array}{c}14,7 \\
(14,0-16,0)\end{array}$ & $\begin{array}{c}16,4 \\
(15,0-17,7)\end{array}$ & $\begin{array}{c}17,6 \\
(16,0-18,6)\end{array}$ & $\begin{array}{c}19,3 \\
(17,0-20,3)\end{array}$ & $\begin{array}{c}20,1 \\
(19,2-21,0)\end{array}$ \\
\hline \multirow{4}{*}{ Lipides } & $1 \mathrm{C}$ & $\begin{array}{c}4,7^{*} \\
(3,2-5,8)\end{array}$ & $\begin{array}{l}3,9^{* *} \\
-\end{array}$ & $\begin{array}{c}3,4^{*} \\
(2,9 \cdot 3,7)\end{array}$ & $\begin{array}{c}4,3 \\
(3,3-5,5)\end{array}$ & $\begin{array}{c}4,2^{* *} \\
(4,0-4,8)\end{array}$ & $\begin{array}{c}6,5 \\
(3,6-10,0)\end{array}$ & $\begin{array}{c}5,6^{*} \\
(4,3-6,9)\end{array}$ \\
\hline & $1 \mathrm{~N}$ & - & $\begin{array}{c}2,5 * * \\
(2,0-4,0)\end{array}$ & $\begin{array}{c}3,0^{*} \\
(2,0-4,0)\end{array}$ & $\begin{array}{c}4,1 \\
(3,0-5,5)\end{array}$ & $\begin{array}{c}4,5^{* *} \\
(3,0-6,1)\end{array}$ & $\begin{array}{c}5,7 \\
(4,8-7,0)\end{array}$ & $5,6^{*}$ \\
\hline & ${ }_{4} \mathrm{C}$ & $\begin{array}{c}5,7^{*} \\
(4,2-6,9)\end{array}$ & $\begin{array}{c}4,7 \\
(3,6-5,2)\end{array}$ & $\begin{array}{c}3,8 \\
(3,5-4,4)\end{array}$ & $\begin{array}{c}4,7 \\
(3,0-10,0)\end{array}$ & $\begin{array}{c}6,5 \\
(5,7-8,0)\end{array}$ & $\begin{array}{c}7,1 \\
3,4-8,7)\end{array}$ & $\begin{array}{c}8,1 \\
(7,0-10,0)\end{array}$ \\
\hline & $4 \mathrm{~N}$ & - & $\begin{array}{c}5,0 \\
(4,0-6,0)\end{array}$ & $\begin{array}{c}6,0 \\
(4,0-8,0)\end{array}$ & $\begin{array}{c}5,0 \\
(4,0-5,6)\end{array}$ & $\begin{array}{c}6,7 \\
(5,3-8,4)\end{array}$ & $\begin{array}{c}6,7 \\
(5,0-8,0)\end{array}$ & $\begin{array}{c}7,2 \\
(6,2-7,7)\end{array}$ \\
\hline \multirow{4}{*}{ Cendres } & $1 \mathrm{C}$ & $\begin{array}{c}1,7 \\
(1,5-1,9)\end{array}$ & 2,1 & $\begin{array}{c}2,4 \\
(2,3-2,5)\end{array}$ & $\begin{array}{c}2,3 \\
(2,0-2,7)\end{array}$ & $\begin{array}{c}3,0 \\
(2,8-3,1)\end{array}$ & $\begin{array}{c}3,1 \\
(3,0-3,3)\end{array}$ & $\begin{array}{c}3,1 \\
(3,1-3,1)\end{array}$ \\
\hline & $1 \mathrm{~N}$ & - & $\begin{array}{c}1,7 \\
(1,0-2,0)\end{array}$ & $\begin{array}{c}2,0 \\
(2,0-2,0)\end{array}$ & $\begin{array}{c}2,3 \\
(1,3-2,8)\end{array}$ & $\begin{array}{c}2 b \\
(2,0-3,0)\end{array}$ & $\begin{array}{c}2,7 \\
(2,0-3,5)\end{array}$ & 2,9 \\
\hline & $4 \mathrm{C}$ & $\begin{array}{c}1,7 \\
(1,4-2,0)\end{array}$ & $\begin{array}{c}1,9 \\
(1,7-2,2)\end{array}$ & $\begin{array}{c}2,3 \\
(2,3-2,4)\end{array}$ & $\begin{array}{c}2,2 \\
(2,0-2,7)\end{array}$ & $\begin{array}{c}2,8 \\
(2,0-3,1)\end{array}$ & $\begin{array}{c}3,0 \\
(2,9-3,3)\end{array}$ & $\begin{array}{c}2,6 \\
(2,0-3,0)\end{array}$ \\
\hline & $4 \mathrm{~N}$ & & $\begin{array}{c}1,5 \\
(1,0-2,0)\end{array}$ & $\begin{array}{c}2,0 \\
(2,0-2,0)\end{array}$ & $\begin{array}{c}2,3 \\
(2,0-2,8)\end{array}$ & $\begin{array}{c}2,8 \\
(2,0-3,0)\end{array}$ & $\begin{array}{c}2,7 \\
(2,0-3,0)\end{array}$ & $\begin{array}{c}3,0 \\
(2,8-3,2)\end{array}$ \\
\hline
\end{tabular}

Entre parenthèses : valeurs extrêmes.

* Différence entre les lots $1(1 \mathrm{C}+1 \mathrm{~N})$ et $4(4 \mathrm{C}+4 \mathrm{~N})$ significatives au seuil 5 p. 100.

** Différence entre les lots $1(1 \mathrm{C}+1 \mathrm{~N})$ et $4(4 \mathrm{C}+4 \mathrm{~N})$ significatives au seuil 1 p. 100 . 
La composition chimique globale dépend de l'âge des animaux. La teneur en matière sèche augmente progressivement entre le $I^{\text {er }}$ et le $28^{\mathrm{e}}$ jour. Cette déshydratation des tissus a pour effet de concentrer tous les composants de la dépouille. Mais, alors que la proportion de protéines s'élève parallèlement à celle des matières sèches, la teneur en cendres s'accroît très rapidement pour atteindre une valeur immuable de $3 \mathrm{p}$. roo après le $I 4^{\mathrm{e}}$ jour. Ces modifications ne sont liées ni à l'origine des poussins, ni au régime qu'ils reçoivent. En excluant les matières grasses, on constate, à la suite de l'un de nous (DELPECH, I966), que la composition corporelle dépend très peu des conditions de vie de l'animal.

A l'inverse des 3 composants que nous venons d'évoquer, les lipides subissent des variations d'ordre quantitatif qui ne sont pas uniquement associées à l'âge du poussin. Dans tous les cas, la proportion de graisse tend quelque peu à diminuer pendant les 2 à 5 premiers jours de vie pour augmenter par la suite; mais il existe des différences entre lots qui sont nettement plus accusées. Le tableau 8 montre qu'à un âge donné, les teneurs moyennes sont toujours plus basses dans les lots I (IN et IC) que dans les lots $4\left(4 \mathrm{~N}\right.$ et $\left.{ }_{4} \mathrm{C}\right)$. Etant donné le faible nombre d'animaux analysés (cf. tabl. 6) et l'importance des fluctuations individuelles qui est de règle pour les lipides, on a groupé les animaux des lots I et 4 pour analyser les résultats statistiquement.

$\gamma)$ Composition en acides gras de divers tissus.

Nous mentionnons seulement les résultats permettant d'apprécier l'état de déficience des animaux en acides gras essentiels, autrement dit, les teneurs en acides linoléique, arachidonique et eïcosatriénoïque. Les autres acides gras sont répartis dans les molécules lipidiques suivant les proportions habituelles et leurs variations n'ont rien de remarquable.

La figure I montre comment évolue la teneur du foie en acides gras polyinsaturés au cours des 28 jours d'expérience.

L'effet du régime maternel est manifeste au moment de la naissance. Après I4 jours de vie, il est à peu près nul; la composition du foie en acides gras paraît alors étroitement liée à celle de 1'aliment distribué au poussin. Témoin de la déficience en acides gras essentiels, l'acide eïcosatriénoïque disparaît très tôt chez les animaux provenant de mères carencées, mais recevant un régime normal (lot IN). D'une façon plus générale, après 1'âge de $\mathrm{I}_{4}$ jours, on distingue seulement deux groupes d'animaux : ceux qui ingèrent de l'acide linoléique (lots IN et $4 \mathrm{~N}$ ) et ceux qui en sont privés (lots $\mathrm{IC}$ et $4 \mathrm{C}$ ).

L'analyse des triglycérides de réserve confirme ce point de vue ; elle fait 1'objet de la figure 2. La teneur en acide linoléique, seul acide gras polyinsaturé présent dans le tissu adipeux en quantité notable, dépend uniquement de l'apport alimentaire lorsque le poussin est âgé de plus de I4 jours.

Les résultats concernant le cerveau sont rapportés dans la figure 3. Le comportement du tissu nerveux s'oppose manifestement à celui du foie et des triglycérides. de réserve. L'influence maternelle demeure marquée jusqu'au $28^{\circ}$ jour d'expérience. Chez les animaux nés d'une mère carencée, la distribution d'un régime normal (lot IN) ne fait pas disparaître l'acide eïcosatriénoïque, alors que dans le foie, cet acide gras est presque entièrement résorbé 4 jours après la naissance. Dans le cas de l'acide arachidonique, 1'influence de 1'apport alimentaire ne devient prépondérant. qu'à la fin de l'expérience. 

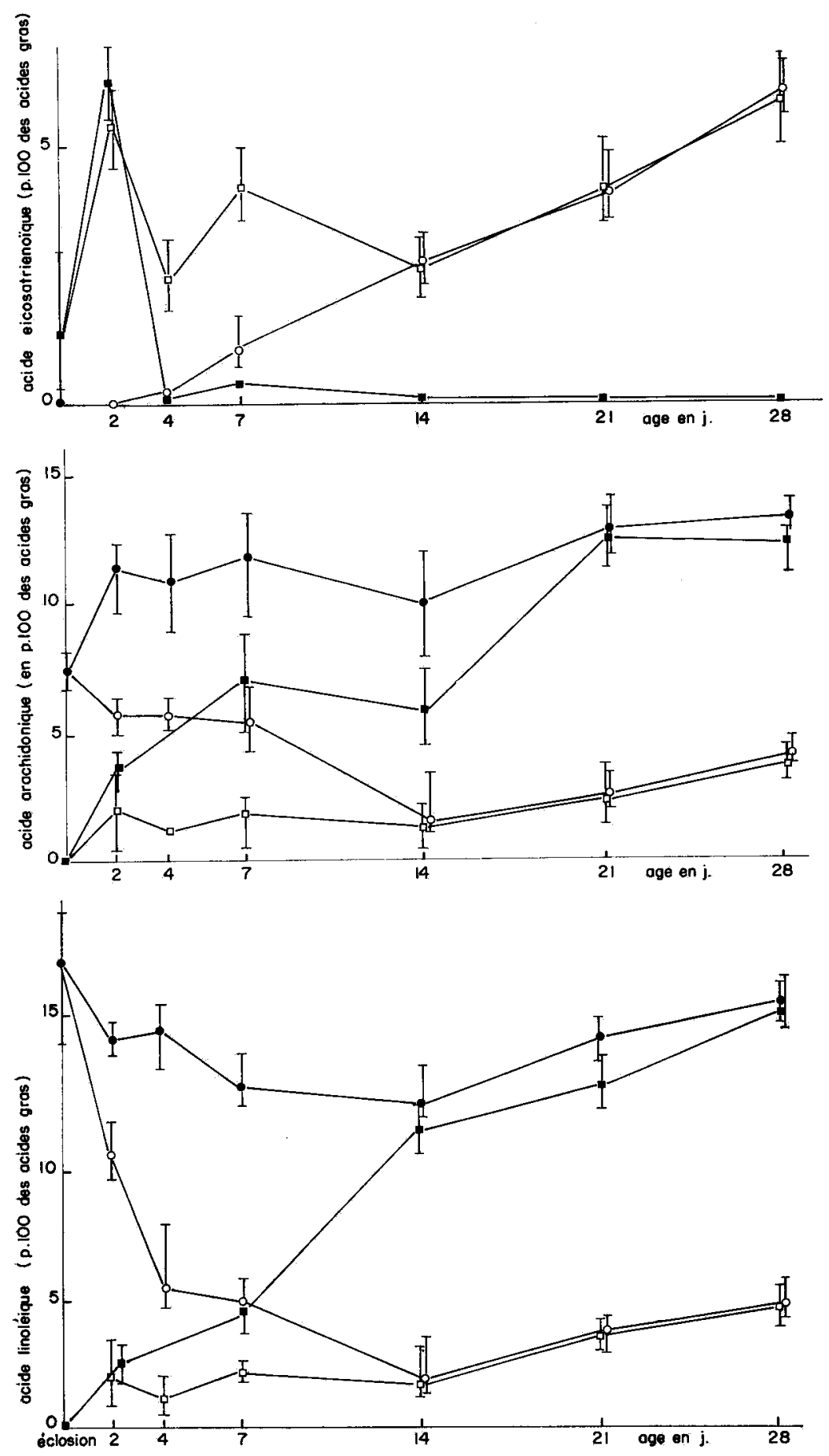

Fig. I. - Evolution de la teneur du foie en acides polyinsaturés : : lot IC $:$ : lot IN $0:$ : lot $4 \mathrm{C} \quad \bullet:$ lot $4 \mathrm{~N}$ 


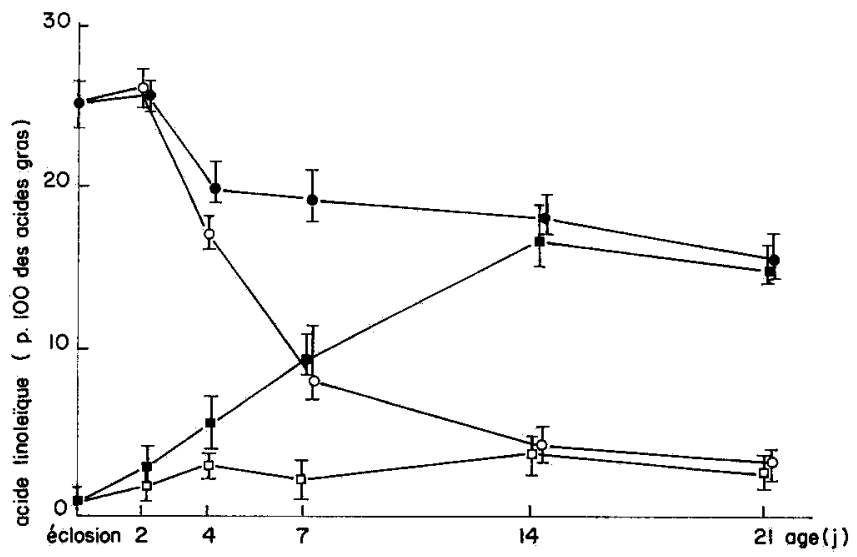

FIG. 2. - Évolution de la teneur des lipides de réserves en acide linoléique
$\square: \operatorname{lot} \mathrm{IC}$
a : $\operatorname{lot} \mathrm{IN}$
$0:$ lot ${ }_{4} \mathrm{C}$
- : lot ${ }_{4} \mathrm{~N}$
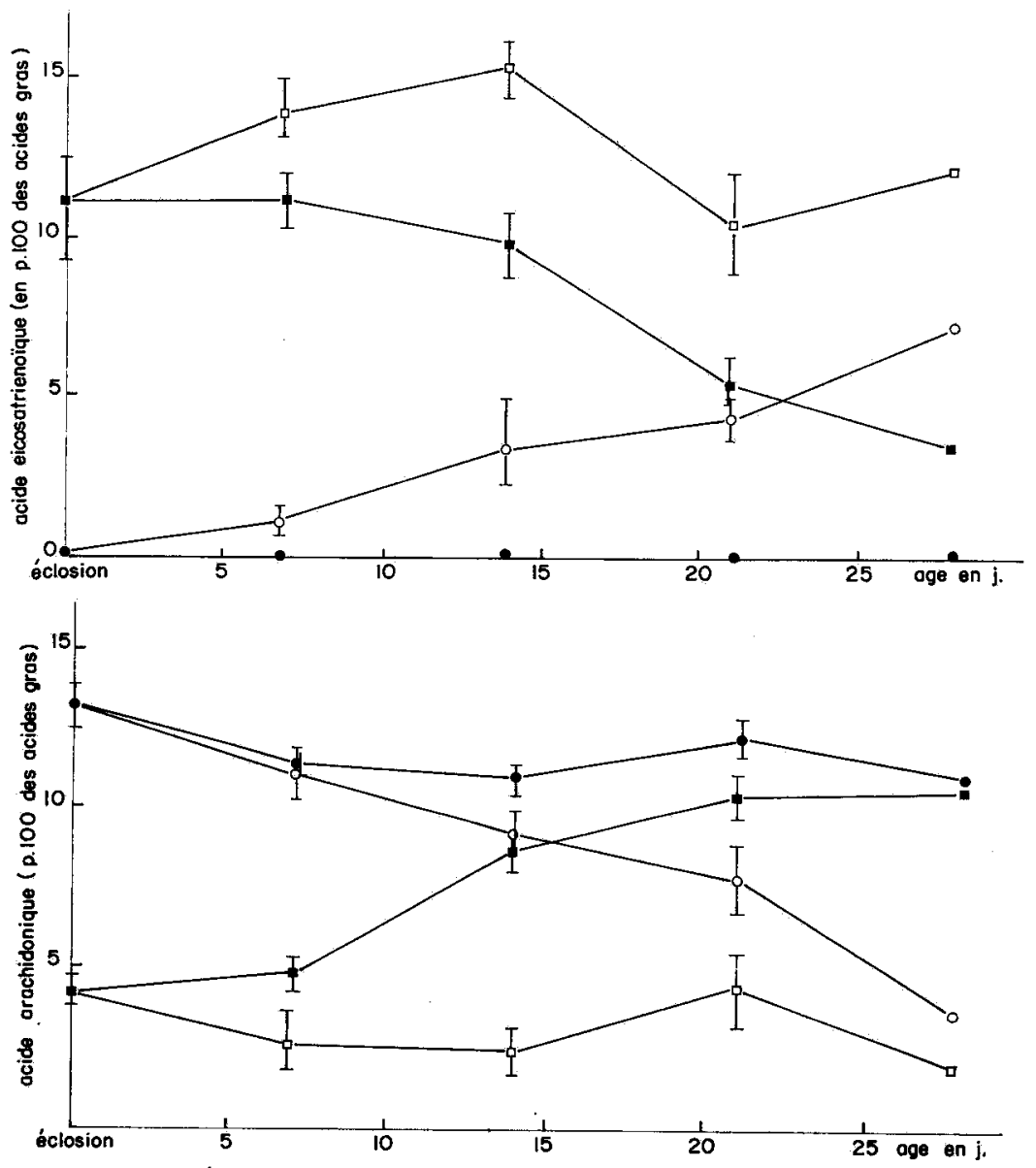

FIG. 3. - Évolution de la teneur des lipides du cerveau en acides polyinsaturés a : lot $\mathrm{IC}, \quad \square: \operatorname{lot} \mathrm{xN} \quad 0: \operatorname{lot} 4 \mathrm{C} \quad \bullet: \operatorname{lot} 4 \mathrm{~N}$

Annales de Biologie animale. - 1969 . 


\section{DISCUSSION}

L'importance des lipides dans l'alimentation de la Poule pondeuse a été soulignée par de nombreux auteurs (JENSEN et SHUTZE, I963; CALVERT, I965). On sait, en particulier, qu'il faut $2,5 \mathrm{p}$. Ioo d'acide liroléique dans le régime pour que le poids de l'œuf atteigne une valeur maximum. Nos résultats confirment cette observation ; en cas de déficience (lot $\mathrm{r}$ ), la taille de l'œuf est réduite de ro p. Ioo.

Par ailleurs, divers travaux ont montré que l'intensité de croissance augmentait en même temps que le poids de 1'œuf (UPP, I928 ; GooDwIN I96I). Notre essai étant réalisé à une époque où le poids de l'œuf ne cesse de s'accroître, nous avons pu rechercher son influence sur le développement du Poussin. Cette influence paraît très faible : chez les animaux témoins (lot $4 \mathrm{~N}$ ), il n'existe aucune corrélation entre le poids de l'œuf et celui du poussin à l'âge de 7 jours. Par conséquent, la lenteur de l'accroissement pondéral chez les poussins nés de mères carencées ne doit pas être uniquenıent attribuée à la taille réduite de l'œuf. En fait, le développement embryonnaire paraît lui-même ralenti ; en l'absence d'acide linoléique, les réserves vitellines sont difficilement résorbées ; elles restent abondantes au moment de la naissance et disparaissent très lentement lorsque le Poussin dispose, dans son régime, d'acides gras essentiels (lot $\mathrm{IN})$.

Si le régime maternel prolonge son effet pendant toute la durée de l'expérience, on peut, cependant, distinguer deux périodes. Pendant la première ( 14 premiers jours), l'influence maternelle est prédominante ; l'indice de consommation est voisin dans tous les lots et 1'intensité de croissance diffère peu selon que l'animal reçoit ou non un régime carencé. Après le $\mathrm{I}_{4}^{\mathrm{e}}$ jour, le développement pondéral dépend davantage de l'aliment ingéré; l'indice de consommation augmente chez les poussins ne recevant pas d'acides gras essentiels et, en ce qui concerne le poids des animaux, si l'écart entre les lots IN et $4 \mathrm{~N}$ consommant le régime normal reste élevé, il tend quelque peu à se combler. Le dosage des acides gras dans divers tissus confirme 1'existence de ces deux périodes. Il faut au Poussin I4 jours de vie pour effacer l'influence du régime maternel au niveau du foie et du tissu adipeux; tandis que le renouvellement des acides gras du cerveau exige un laps de temps supérieur à toute la durée de 1'expérience. Ainsi, chez le Poussin âgé de plus de I4 jours, seuls certains constituants corporels (ou cellulaires) conservent des acides gras déposés dans l'œuf aut cours de l'ovogenèse. Il est difficile d'édifier des hypothèses sur la nature de ces constituants ; mais étant donné ce que l'on sait de la structure et du rôle des membranes, on peut supposer que leur influence s'exerce au niveau des échanges cellulaires qui contrôlent l'utilisation des nutriments et, partant, l'intensité de croissance.

Ce mode d'intervention des acides gras essentiels rend compte de nos observations montrant que la croissance reste harmonieuse. Chez les animaux carencés, la proportion des divers constituants corporels n'est pas modifiée ; il n'y a pas de stéatose hépatique, ce qui va à l'encontre des résultats obtenus par Hopkins (I964) chez des animaux plus âgés. Dépendant surtout de l'âge des animaux (DEL,PECH, Ig66) les proportions respectives d'eau, protéines et cendres ne sont pas modifiées par la déficience en acide linoléique, qu'elle intervienne avant (régime maternel) ou après 1'éclo- 
sion (régime du poussin). Au contraire, l'aptitude à l'engraissement qui varie selon. les individus, semble profondément altérée lorsque les animaux sont issus de mères. carencées en acides gras essentiels. Force est d'admettre que certains processus biochimiques responsables de la formation du tissu adipeux sont mis en place au cours de l'embryogenèse et subsistent longtemps après la naissance. Seul l'usage d'éléments marqués pourrait indiquer comment intervient la déficience du régime maternel : diminution de la vitesse de synthèse ou bien utilisation accrue des dépôts lipidiques. Cette dernière hypothèse pourrait trouver un appui dans les essais effectués in vitro sur du tissu adipeux de rat par BERGSTRöm et CARLSON (I965). Selon ces auteurs, la déficience en acides gras essentiels accélère la mobilisation des lipides; il est possible que cette action provienne d'un défaut de formation des prostaglandines qui sont, on le sait, synthétisées à partir des acides gras polyinsaturés.

\section{CONCLUSION}

Antérieurement à cet essai, quelques travaux ont montré qu'une déficience du régime maternel en acide linoléique était susceptible de ralentir la croissance du poussin. Mais on pouvait supposer que cet effet était analogue à celui d'une carence survenant au cours de la vie post-natale. Nos résultats montrent que la déficience du régime maternel intervient suivant des modalités distinctes : influence très marquée sur le développement pondéral du poussin, diminution de l'aptitude à l'engraissement. Cependant, nous tenons à souligner que ces conclusions ne concernent que des animaux très jeunes ingérant des quantités d'acide linoléique habituellement suffisantes, mais non pléthoriques. Dans d'autres conditions expérimentales, l'effet maternel pourra être nul ou peu apparent (HopkINs, I964). On doit supposer, selon toute vraisemblance, que la vie de l'animal n'est pas définitivement perturbée par le régime maternel. Nos observations révèlent seulement que les conditions qui président au développement de l'embryon ont un rôle particulièrement important sur le devenir ultérieur de l'animal. C'est donc là un des aspects de l'influence du jeune âge sur la vie future. Plus une déficience alimentaire intervient tôt dans la vie de l'animal, plus ses effets sur la croissance sont prononcés (SrNGSEN et al., I964). Nos résultats semblent bien confirmer ce point de vue puisque l'on parvient plus facilement à modifier les processus qui règlent la croissance en carençant l'animal au cours de sa vie embryonnaire plutôt que pendant sa vie post-natale.

Rę̧u pour publication en janvier 1969.

\section{SUMMARY}

INFLUENCE OF MATERNAL DIET ON THE GROWTH OF YOUNG CHICKENS.

EFFECT OF DEFICIENCY OF LINOLEIC ACID

Forty-eight Cornish hens were divided into 4 groups according to the linoleic acid content of the diet : $0,1,2$ or 5 per cent. The chick hatched from eggs laid by these hens were given a feed with o.I or I.I per cent linoleic acid as maize oil. They were killed at ages between 0 and 28 days. Gain and daily intake of feed were recorded. Analyses were made of separation of body constituents (liver, 
brain, skin and feathers, carcass...), estimation of protein, lipids and ash, extraction and estimation of fatty acids in liver, carcass and brain.

When the hens had been given the feed deficient in linoleic acid weight of the egg and of the newly hatched chick were both reduced. Growth after hatching was retarded ; a supply of $I . I$ per cent linoleic acid in the diet of the chick did not allow resumption of normal growth during the 28 days of the experiment.

Nevertheless growth remained harmonious. Contents of protein and ash depended only on the age of the chick. In contrast, deposit of fat was related to the content of the maternal diet in essential fatty acids ; whichever feed was given to the chickens, those hatched from deprived mothers were leaner than those from control hens.

At hatching the fatty acid composition of all the tissues was closely related to that of the maternal diet. However, in liver and carcass the formation was rapid and after I4 days of age the fatty acid composition depended only on the diet of the chick.

Lipids in brain, on the other hand, remained " marked " by the maternal diet for much longer and at 28 days of age this effect was still accentuated.

Deficiency of linoleic acid in the egg from the effect of maternal diet allows an explanation of the slowing of growth of the chick. The effects of the deficiency are the greater the earlier they are applied in the course of development.

\section{RÉFÉRENCES BIBLIOGRAPHIQUES}

Bergström S., Carlson L. A., 1965. Lipid mobilization in essential fatty acid deficient Rats. Acta physiol. Scand., 64, 479-480.

Calvert C. C., 1965. Lipid metabolism in the laying hen : essential fatty acids. Proc. Cornell Nutr. Cont. Feed Manuf., November, 2-3-4, 104-1 22.

DetPeCH P., I 966 . Le poids frais délipidé chez Gallus Gallus : relations qui unissent ses constituants. $\quad C . R$. Acad. Sci., 203, 1735-1 738.

DelPech P., Guezel M., Leclerce B., Kahane E., 1966. Méthode d'extraction des lipides en continu et à chaud par le mélange azéotrope benzène-éthanol-eau. Rev. Fr. Corps Gras, 10, 6I 5-620.

DONALDSON W. E., 1967. Lipid composition of chick embryo and yolk as affected by stage of incubation and maternal diet. Poultry Sci., 46, 693-697.

Goobwin K., J95r. Effect of hatching size and chick size upon subsequent growth rate in chicken. Poultry Sci., 40, I 408 .

Hopkins D. T., Nesherm M. C., ig62. The effect of linoleic acid depletion on performance of hens and their progeny. Poullry Sci., 41, 165 .

Isaaks R. E., Davies R. E., Ferguson T. M., Reiser R., Couch J. R., Ig64. Studies on avian fat composition. 2. Selective utilization of fatty acids by the chick embryo. Poultry Sci., 48, I I 3-1 20.

LecLerce B., 1966. Contribution à l'étude du métabolisme des lipides chez l'embryon de poulet : utilisation des réserves vitellines en présence d'acide élaìdique incorporé dans l'œeuf par la poule pondeuse. $A n n$. Biol. anim. Bioch. Biophys., 6, 503-516.

Menge H., Miller E. C., Denton C. A., 1964. Effect of fat-free maternal diet on fatty acid composition of progeny. Poultry Sci., 43, 164-168.

Singsen E. P., Nagel J., Patrick S. G., Matterson L. D., 1964. The effet of a lysine deficiency on body weight and age at sexual maturity of meat type pullets. Pouliry Sci, 43, 786-788.

UPP C. W., 1928. Egg weight, day-old chick weight and rate of growth in single comb Rhode Island Red chicks. Poullyy Sci., 7, I5 1-155. 